\title{
Robotic Joint Torque Testing: A Critical Tool in the Development of Pressure Suit Mobility Elements
}

\author{
Forrest E. Meyen ${ }^{1}$ \\ University of Missouri, Columbia, Missouri, 65201 \\ Bradley Holschuh ${ }^{2}$, Ryan L. Kobrick ${ }^{3}$ \\ Massachusetts Institute of Technology, Cambridge, Massachusetts, 02139 \\ Shane E. Jacobs ${ }^{4}$ \\ David Clark Company, Worcester, Massachusetts, 01604 \\ Dava J. Newman ${ }^{5}$ \\ Massachusetts Institute of Technology, Cambridge, Massachusetts, 02139
}

\begin{abstract}
Pressure suits allow pilots and astronauts to survive in extreme environments at the edge of Earth's atmosphere and in the vacuum of space. One obstacle that pilots and astronauts face is that gas-pressurized suits stiffen when pressurized and greatly limit user mobility. As a result, a critical need exists to quantify and improve the mobility characteristics of pressure suits. A historical survey and critique of pressure-suit testing methodologies is first presented, followed by the results of recent pressure suit testing conducted at the MIT Man-Vehicle Laboratory (MVL). MVL researchers, in cooperation with the David Clark Company (Worcester, MA), used an anthropometrically-realistic robotic space suit tester to quantify pressure suit mobility characteristics of the S1034 Pilot Protective Assembly (PPA), a pressure suit worn by $\mathrm{U}-2$ pilots. This suit was evaluated unpressurized, at a vent pressure of $5.5 \mathrm{kPa}(0.8 \mathrm{psi})$, and at an emergency gauge pressure of $20.7 \mathrm{kPa}(3 \mathrm{psi})$. Joint torque data was collected for elbow flexion/extension, shoulder flexion/extension, shoulder abduction/adduction, and knee flexion/extension motions. The aim of this study was to generate a robust baseline mobility database for the S1034 PPA to serve as a point of comparison for future pressure suit designs, and to provide recommendations for future pressure garment testing.
\end{abstract}

\section{Introduction}

$\mathrm{P}$ ressure suits are worn by pilots and astronauts to protect them from low pressure environments at the edge of the atmosphere and in the vacuum of space. These suits are typically worn in situations where the user needs to be mobile enough to complete a task that is not possible from a pressurized vehicle. Even though pressure suits such as NASA's Extravehicular Mobility Unit (EMU) were designed for tasks such as space walks during the assembly of the International Space Station, the EMU, like all gas pressurized suits, is restrictive to user mobility ${ }^{1}$. This fact has been observed since pressure suit development was in its absolute infancy ${ }^{2}$.

\footnotetext{
${ }^{1}$ Undergraduate, Mechanical and Aerospace Engineering, 18932 Twenty Grand Road, Eagle River AK 99577

${ }^{2}$ Ph.D. Candidate, Aeronautics and Astronautics, 77 Massachusetts Avenue Room 37-219, AIAA Student Member

${ }^{3}$ Postdoctoral Associate, Aeronautics and Astronautics, 77 Massachusetts Avenue Room 37-219, AIAA Member

${ }^{4}$ Design Engineer, Research and Development, David Clark Company, AIAA Member

${ }^{5}$ Professor, Aeronautics and Astronautics and Engineering Systems, 77 Massachusetts Avenue Room 33-307, AIAA Associate Fellow
} 
The resistance of a pressurized suit to bending, hereafter referred to as the joint torque, is the result of three different effects: structural, volume, and pressure effects. Structural effects are caused by the stiffening of suit materials when pressurized. Volume effects result from changes in internal suit volume during joint articulation (i.e., the work needed to complete an ideal, isobaric compression) $)^{3}$. Pressure effects represent additional resistance to movement caused by changes in internal pressure when the suit volume is decreased (i.e., the additional work, above and beyond that required for ideal isobaric compression, spent because suit pressure changes during internal volume change). Pressure effects are caused by volume effects, but are a separable effect. In most pressure suit joint designs, volume effects make up the majority of the joint torque, dominating the structural effects. Pressure effects are typically negligible, as pressure suit Life Support Systems maintain pressure precisely. A good description of the pressurized volume effects, and rough calculation of the torque required to bend variously sized joints given no design features, is provided by Harris ${ }^{4}$.

As NASA prepares for ambitious exploration missions outside of low earth orbit, it is increasingly important to be able to quantify, communicate, and validate space suit mobility. Throughout various space suit development projects, several methodologies of joint torque measurement have been explored. Many have been used by suit designers to track their progress in their quest to reduce torque ${ }^{5-9}$. One of the long term goals of pressure suit design is to reduce the induced joint torques caused by the suit, eliminating work done by the subject to move the suit, so that all of the subject's energies can be spent accomplishing the tasks at hand. In order to achieve this goal, the torques induced by various joint designs must be well understood and quantified. Improvements to the joints can then in turn be quantified, by measuring the reduction in induced torque, and understanding the physical principles responsible for the reduction. Without continuous benchmarking and iteration, the suit designer cannot make progress towards a zero (or near zero) torque joint.

Recent joint torque quantification projects have aimed to quantify torques for the purposes of writing requirements ${ }^{10}$, while others have intended to quantify and understand the various components of joint torque with the ultimate end goal of creating predictive models ${ }^{11}$. Understanding and predicting exactly how much torque a crewmember must exert to perform each task would enable planning and modeling of tasks to reduce crewmember fatigue, maintain consumables, develop reasonable and obtainable schedules and maintain low metabolic rates.

The most widespread and traditional means of measuring joint torque is to externally bend the joint manually, ensuring it remains in the horizontal plane to eliminate gravity effects, measuring the load it takes to bend the joint, and calculating the resulting torque. In the early days of suit development a fish scale was used to measure the applied load at the end of the joint. The torque is simply the cross product of the moment arm and the force measured by the fish scale. Provided that the person performing the test pulls the joint along a path perpendicular to the moment arm throughout the rotation of the joint, the torque can be calculated by simply finding the product of the force on the fish scale and the distance between the center of rotation of the joint and the point the force was applied. Concurrently measuring the joint angle using a protractor or other means, and recording values of joint torque for many angles throughout the full rotation of the joint provides the data to create the traditional torque vs. joint angle curve. The fish scale method is shown in Figure 1a, in which engineers are measuring the joint torque on an early pressure suit, designed for the Air Force Manned Orbiting Laboratory program ${ }^{5}$. This program was later cancelled, but this picture shows perhaps the earliest measurements of pressure suit joint torque. Similar methods were used in the development of the Apollo suits ${ }^{6}$.

As technology has developed, the fish-scale method has been upgraded to provide higher repeatability and greater precision. The fish scale itself has been replaced by a small inline load cell, and the protractor replaced with rotary encoders mounted to the center of rotation of the joint, or other digital means of measuring angles such as multiple accelerometers, with each sensor feeding data into a data acquisition system. This provides high-bandwidth data, direct synchronization of the data streams, and significantly reduces operator subjectivity as a source of error. The digital capture of the data also enables dynamic measurements of joint torque, whereas the fish-scale method is limited to quasi-static measurement.

Joint torques have been measured in both manned and unmanned suits, and using both positive pressure (i.e., the interior of the suit is pressurized) and negative pressure (i.e., the exterior of the joint is exposed to vacuum) test configurations (see Figures 1b-c). Each method has its own unique advantages and disadvantages. Unmanned measurements have the advantage of minimizing anthropometric variables, uncertainties, and inconsistencies between subjects. However, as the largest component of the torque is from pressurized volume effects ${ }^{3}$, and as the human body takes up most of the free volume within a space suit thereby drastically reducing the total volume that can potentially change during joint movement, it is unclear how realistically an unmanned joint torque measurement predicts the manned joint torque. The exact total volume occupied by the robot is not known, but it is comparable in size to a $1.9 \mathrm{~m}$ tall human. In human subjects, there is great variation in occupied volume within the suit. Quantifying the volume of the robot in relation to the statistical variation of human volumes is recommended. 

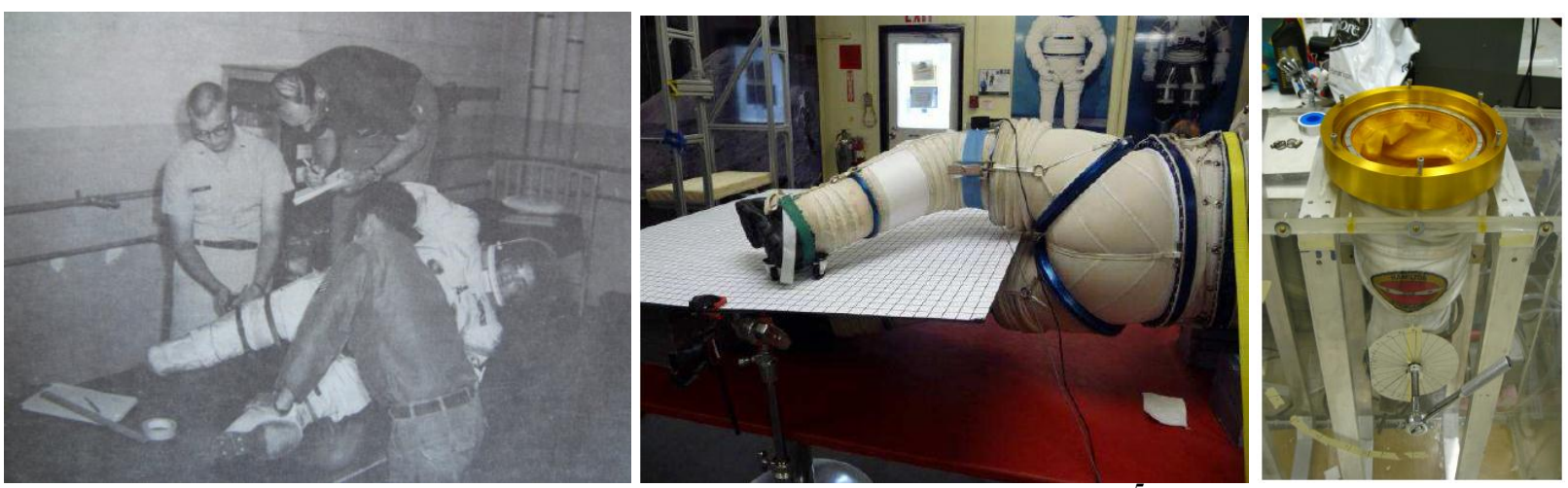

Figures 1a-c. Example of early pressure suit joint torque measurement (left) ${ }^{5}$, positive pressure tabletop testing $(\text { center })^{14}$, and negative pressure vacuum chamber testing (right) ${ }^{3}$

Additionally, in a manned joint, the human body comes in contact with the space suit internally during joint motion, which affects how the joint bends. An unmanned joint can shift its axis in ways that a manned joint cannot. This can cause erroneous data for the joint torque when measuring it unmanned, though this is heavily dependent on the joint design (some joint designs may not exhibit this difference while in others it may be substantial). Performing the fish scale measurements manned, as shown in Figure 1a, may not necessarily provide any more accurate data than unmanned. When measuring manned torques, the subject must attempt to minimize his/her effect on the joint torque by not aiding or restricting the bending of the joint. This introduces a very complex potential source of uncertainty.

Another method that has been used to quantify the torques of a suit, in this case the EMU, was to compare suited and unsuited strengths of human subjects using a dynamometer to provide resistance as the subjects moved through their comfortable range of motion. This method provided good data about suited astronaut strength, but the data was not detailed enough to show the hysteretic characteristics of joint torque movement ${ }^{12}$. Hysteresis is cyclical energy loss that occurs throughout a cycle and is a commonly observed property of space suit joints ${ }^{11}$.

An alternative to these methods is to collect suit torque data using robotic systems, such as MIT's Man-Vehicle Laboratory (MVL) Robotic Space Suit Tester (RSST). The RSST, pictured in Figure 2a, is a hydraulically actuated robot designed by NASA and built by Sarcos Inc., and was originally designed to quantify joint torques for advanced space suit mobility development. NASA donated the RSST to MIT for use in support of student research projects. The RSST has 12 articulating joints on its right side, and each joint contains a potentiometer to measure joint angle and a strain gauge load cell to measure joint torque. During the early 2000s the RSST was used extensively by the MVL to create joint torque database for the EMU ${ }^{13}$.

This robotic system has its own set of advantages and disadvantages. The first main advantage is that the robot replicates the interior 'free-volume' volume of suit in a similar manner to that of a human subject, but without the associated anthropometric variation of an actual human. The robot's anthropometry is fixed and quantifiable, and its motions are exactly repeatable. It reduces the free volume interior to the suit, thereby more closely approximating the actual volume change during joint motion, as compared to unmanned methodologies. It also interacts with the interior of the suit, preventing the axis of the joint from shifting in non-natural ways. However, these interactions are not necessarily identical to those that would occur with an actual human subject. Another main advantage of the RSST is that it measures the torque required to bend the joint from the inside of the suit, which is a better approximation of how the suit subject bends the joint. The other methodologies, whether manned or unmanned, are measuring an external torque, which is not exactly representative of the biomechanical process used to bend the joint internally.

The RSST has some drawbacks that prevent a direct correlation between measured joint torques using the robot and actual suited joint torques. Notably, the robot is not precisely representative of the human form, thus it does not fill the suit in the same way that a human would. The RSST was designed to mimic joint movements but not the surfaces of the human body. Areas that are curved on a human are sometimes sharp and unnatural in the RSST. The RSST does not move in exactly the same manner because the joints were represented with hydraulic actuators and do not match the complex movements of many human joints. Also, the RSST does not interact with the suit internally in exactly the same way. Human muscle can deform when pressed against the walls of the suit while steel robot components will not. These effects are not fully understood, though they should be recognized and evaluated in future work. 
To demonstrate the capabilities of robotic space suit testing, the methods and results of a recent pressure suit mobility study conducted at MIT are included in the following sections.

\section{Methods}

A series of pressure suit mobility tests aimed at quantifying the mobility characteristics of the S1034 Pilot Protective Assembly (PPA) were conducted at the MIT MVL in the summer of 2010. The S1034 PPA is a pressure suit worn by pilots of the U-2, a high altitude reconnaissance airplane operated by the United States Air Force. The suit is normally worn at a vent gauge pressure of $0.7 \mathrm{kPa}(0.1 \mathrm{psi})$ and pressurizes to $24 \mathrm{kPa}(3.5 \mathrm{psi})$ if there is a loss of cabin pressure or when the pilot ejects from the aircraft ${ }^{15,16}$. The S1034 PPA is intended to be worn by seated pilots in the cockpit, at such low differential pressure that the suit is essentially unpressurized. The suit is not designed to provide significant pressurized mobility, as mobility limitations are only of concern in the event of ejection or cabin depressurization when the suit pressure is increased. The PPA is primarily a comfortable and lightweight garment that is also capable of providing protection in the event of an emergency.

The S1034 PPA was donned on the RSST (see Figure 2b), and the torque responses of four joint movements were assessed: elbow flexion/extension, shoulder flexion/extension, shoulder abduction/adduction, and knee flexion/extension. The suit was connected to an air compressor and three pressure conditions were tested: unpressurized $(0 \mathrm{kPa})$, near emergency pressurization $(20.7 \mathrm{kPa})$, and a possible vent pressure for suits used in future exploration vehicles $(5.5 \mathrm{kPa})$. A near emergency pressurization of $20.7 \mathrm{kPa}$ was used instead of the emergency pressurization of $25 \mathrm{kPa}$ to reduce the risk of rupturing the suit during motions it was not designed to accommodate. Each joint was driven through a controlled range of motion envelope, and these envelopes were determined on a case by case basis for each joint and pressure condition to ensure the suit was never driven beyond safe limits. Table 1 details the angle envelopes tested for each joint and each pressure condition. Unsuited torque data was also collected so that gravity effects and inherent robotic resistance could be subtracted from the final data.

Table 1: Range of motion test envelope for each joint and pressure condition

\begin{tabular}{|c|c|c|c|}
\hline Joint Movement & $\begin{array}{l}\text { Test 1: Suit } \\
\text { Unpressurized }\end{array}$ & $\begin{array}{c}\text { Test 2: Predicted Orion } \\
\text { Vent Pressure } \\
5.5 \mathrm{kPa}(0.8 \mathrm{psi}) \\
\end{array}$ & $\begin{array}{l}\text { Test 3: Pressurized } \\
20.7 \mathrm{kPa}(3.0 \mathrm{psi})\end{array}$ \\
\hline Elbow Flexion/Extension & $0^{\circ}$ to $80^{\circ}$ & $0^{\circ}$ to $80^{\circ}$ & $5^{\circ}$ to $45^{\circ}$ \\
\hline Shoulder Flexion/Extension & $-20^{\circ}$ to $60^{\circ}$ & $-15^{\circ}$ to $60^{\circ}$ & $-10^{\circ}$ to $60^{\circ}$ \\
\hline Shoulder Abduction/Adduction & $0^{\circ}$ to $105^{\circ}$ & $5^{\circ}$ to $100^{\circ}$ & $10^{\circ}$ to $80^{\circ}$ \\
\hline Knee Flexion/Extension & $5^{\circ}$ to $95^{\circ}$ & $5^{\circ}$ to $95^{\circ}$ & $10^{\circ}$ to $95^{\circ}$ \\
\hline
\end{tabular}



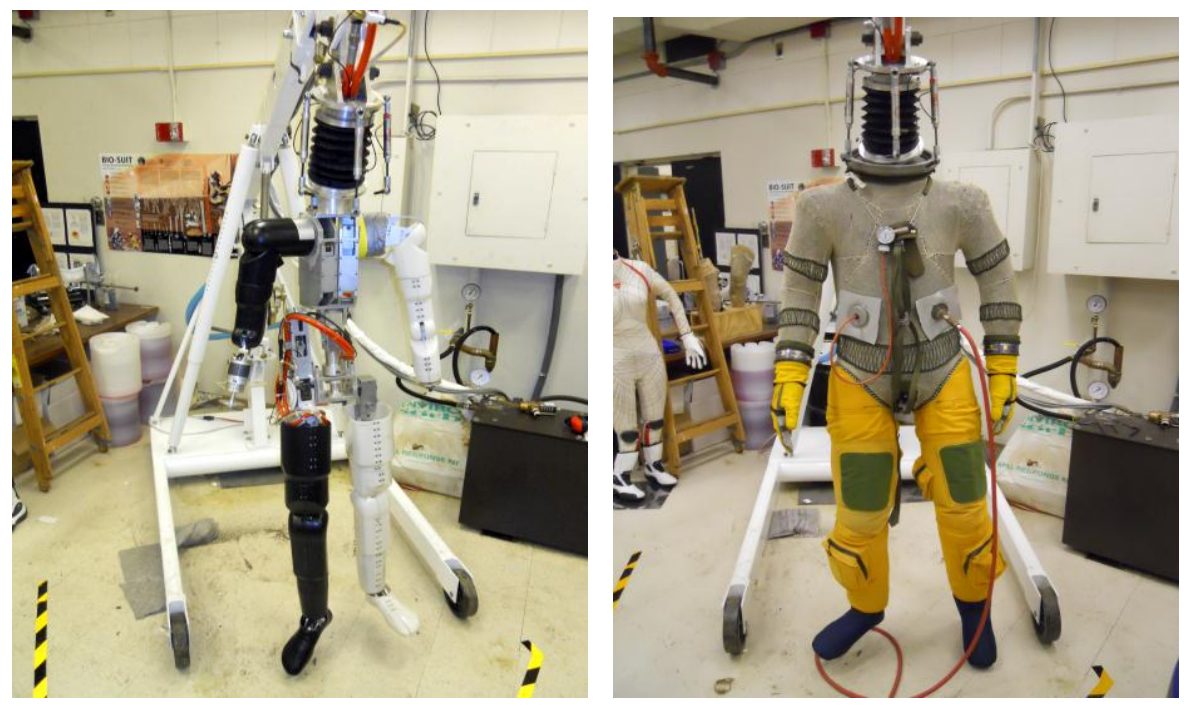

Figures 2a-b. MIT robotic space suit tester unsuited (left), and donned with the S1034 PPA (right)

Torque and position data are generated by the robot test system at $1000 \mathrm{~Hz}$, and the data was collected and analyzed using MATLAB. Elbow flexion/extension movements were controlled by Simulink-generated sinusoidal inputs to the RSST control system. Manual inputs into the RSST control system were used for the shoulder flexion/extension, shoulder abduction/adduction, and knee flexion/extension tests. Because the S1034 PPA was not designed for high pressurized mobility, caution was exercised to ensure the suit was not driven to unsafe joint angles. Manual inputs allowed the operator to easily adjust the speed and range of movement to safe values depending on testing observations. Each movement was sinusoidal and repeated three to six times during a 60 second data collection period.

To control for the effects of the robot weight and hydraulic hysteresis, unsuited data was first recorded for each movement. For each dataset, torque averages at five degree increments were calculated. The unsuited torque averages at each position were subtracted from the average suited torque to remove the torque inherent to the testing system. Figure 3 shows the unsuited (gravity effects) data, original (uncalibrated) torque data, and reduced data for a sample flexion/extension measurement at $20.7 \mathrm{kPa}$. Error bars on each data point indicate a $95 \%$ confidence interval. In many cases the confidence interval is too small to be visible. Larger confidence intervals were noted at some points, possibly because of shifting in the suit fabric as the joint was actuated. All subsequent data presented has been reduced in this manner. 


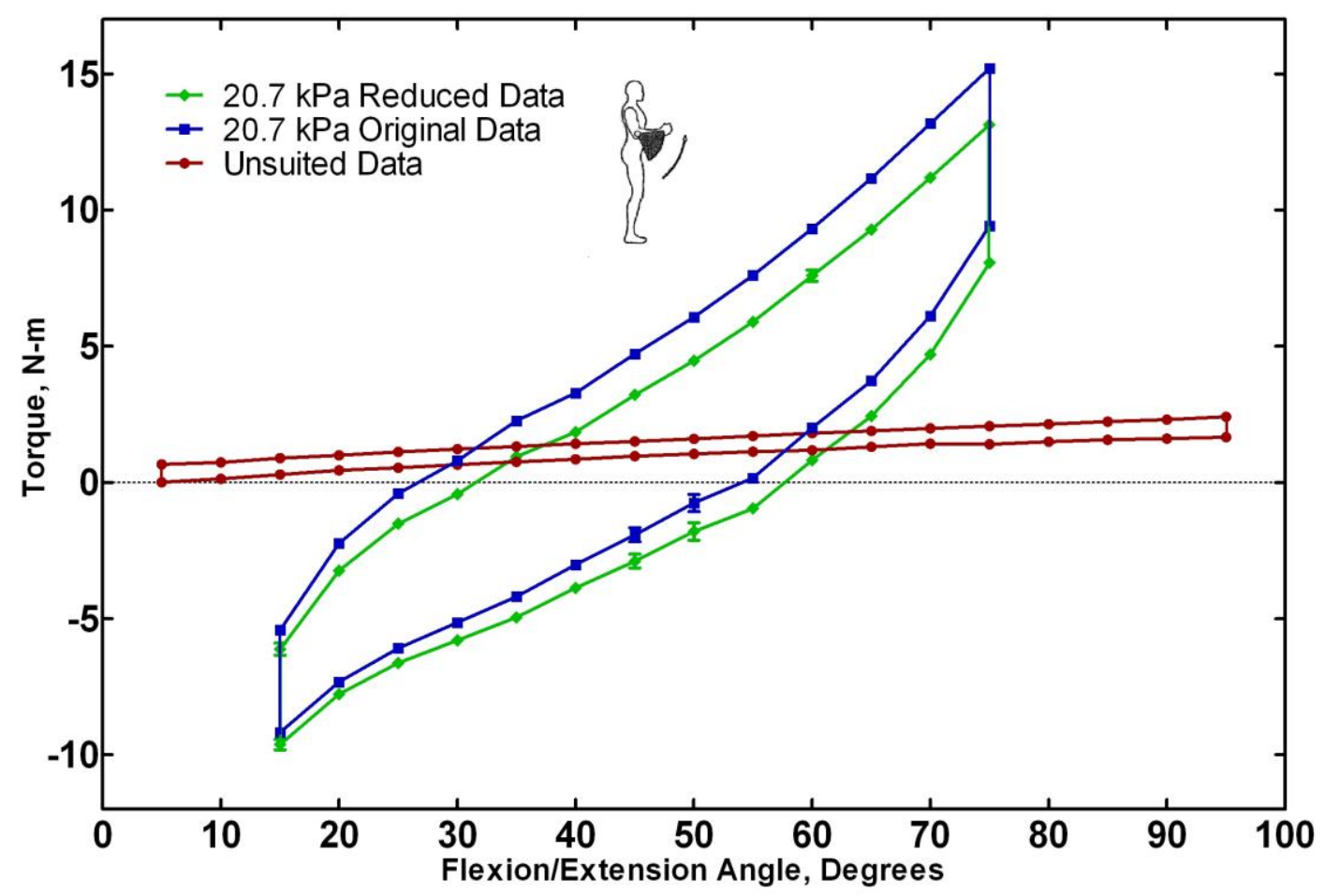

Figure 3. Data calibration to remove gravity effects

\section{Results}

Torque vs. angle data from each joint test are included in Figures 4-7 with gravity effects removed. Confidence intervals for the $95^{\text {th }}$ percentile are presented for each dataset. In each case, hysteresis effects were observed, and the magnitude of these effects increased non-linearly with operating pressure. The elbow flexion/extension dataset contained very little noise (as evidenced by the included confidence intervals which averaged less than $1 \mathrm{~N}-\mathrm{m}$ ) because the input to the robotic motions was controlled by an automatic sinusoid function. All other datasets were collected using manual control inputs, in some cases resulting in noisier data (as evidenced by the larger confidence intervals).

In each case, peak torques were observed at the extreme end of the range of motion envelope, and these torques increased non-linearly as internal pressure increased. For comparison, the torque required to bend each joint to $70^{\circ}$ under each pressure condition is summarized in Table 2 (except shoulder flexion/extension, where torques were compared for $50^{\circ}$ flexion). 
Table 2: Torque comparison for each test condition

\begin{tabular}{|c|c|c|c|}
\hline Joint Movement & $\begin{array}{l}\text { Test 1: Suit } \\
\text { Unpressurized }\end{array}$ & $\mid \begin{array}{cc}\text { Test 2: } & \text { Predicted } \\
\text { Vent Pressure } & 5.5 \\
\text { kPa }(0.8 \mathrm{psi}) & \end{array}$ & $\begin{array}{l}\text { Test 3: Pressurized } \\
20.7 \mathrm{kPa}(3.0 \mathrm{psi})\end{array}$ \\
\hline Elbow Flexion/Extension & $1.32 \mathrm{Nm}$ at $70^{\circ}$ & $4.23 \mathrm{Nm}$ at $70^{\circ}$ & $11.19 \mathrm{Nm}$ at $70^{\circ}$ \\
\hline Shoulder Flexion/Extension & $3.10 \mathrm{Nm}$ at $50^{\circ}$ & $12.44 \mathrm{Nm}$ at $50^{\circ}$ & $24.97 \mathrm{Nm}$ at $50^{\circ}$ \\
\hline Shoulder Abduction/Adduction & $4.39 \mathrm{Nm}$ at $70^{\circ}$ & $17.49 \mathrm{Nm}$ at $70^{\circ}$ & $\mathrm{N} / \mathrm{A}$ \\
\hline Knee Flexion/Extension & $0.84 \mathrm{Nm}$ at $70^{\circ}$ & $6.16 \mathrm{Nm}$ at $70^{\circ}$ & $18.47 \mathrm{Nm}$ at $70^{\circ}$ \\
\hline
\end{tabular}

The resistive torque measured during knee flexion was the greatest of all the joints tested: $36.2 \mathrm{~N}-\mathrm{m}$ was generated when the knee was flexed to $91^{\circ}$ at $20.7 \mathrm{kPa}$. Though this was the highest torque value measured, it is important to note that this does not necessarily mean that the knee joint is the stiffest joint, because each joint was only driven to angles deemed safe by the team based on observations during testing. Other joints may have exhibited higher torque values if they were allowed to be driven to comparable angles (i.e., not all joints were driven to $91^{\circ}$ ).

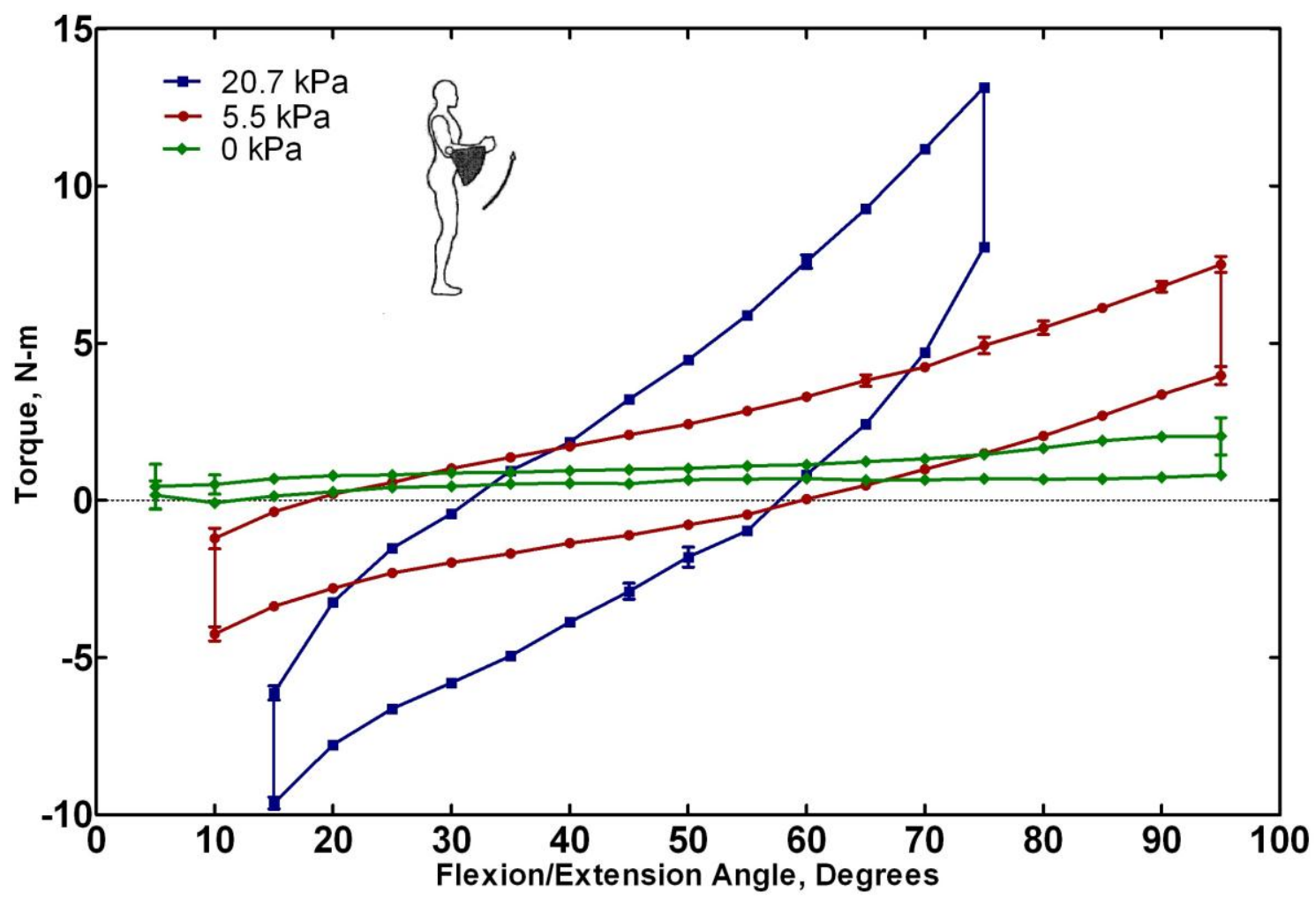

Figure 4. Elbow flexion/extension torque vs. angle data with gravity effects removed 


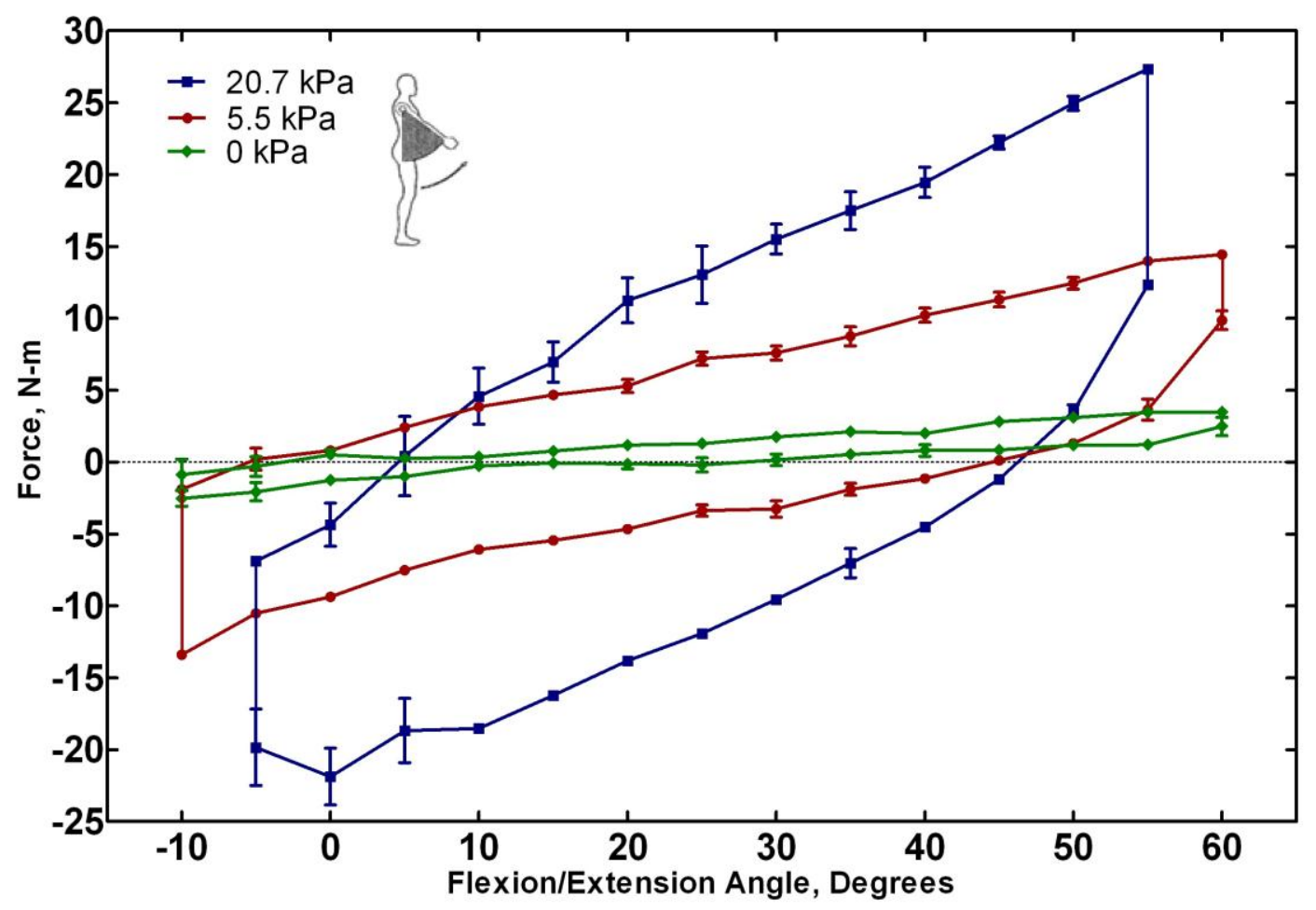

Figure 5. Shoulder flexion/extension torque vs. angle data with gravity effects removed

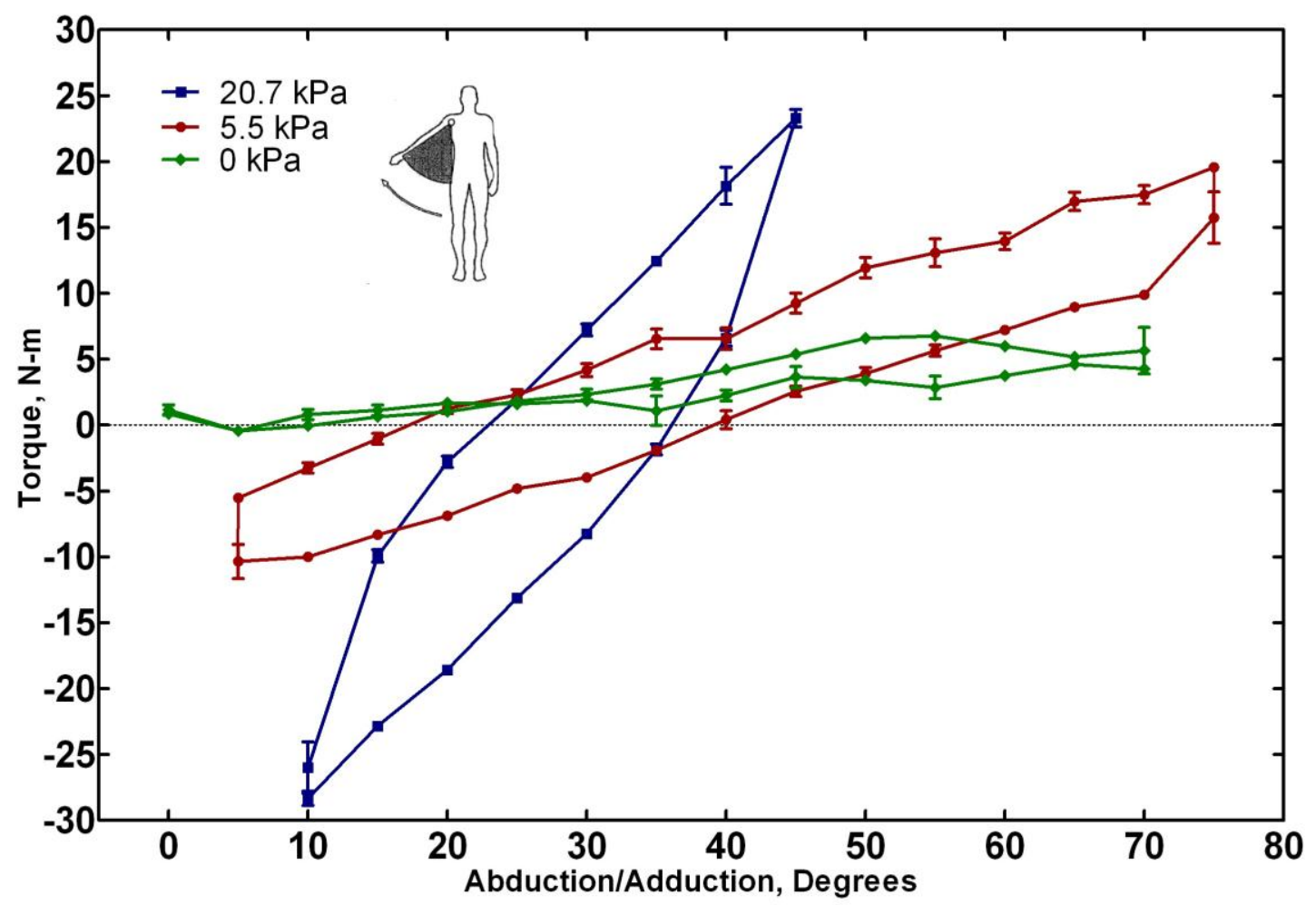

Figure 6. Shoulder abduction/adduction torque vs. angle data with gravity effects removed 


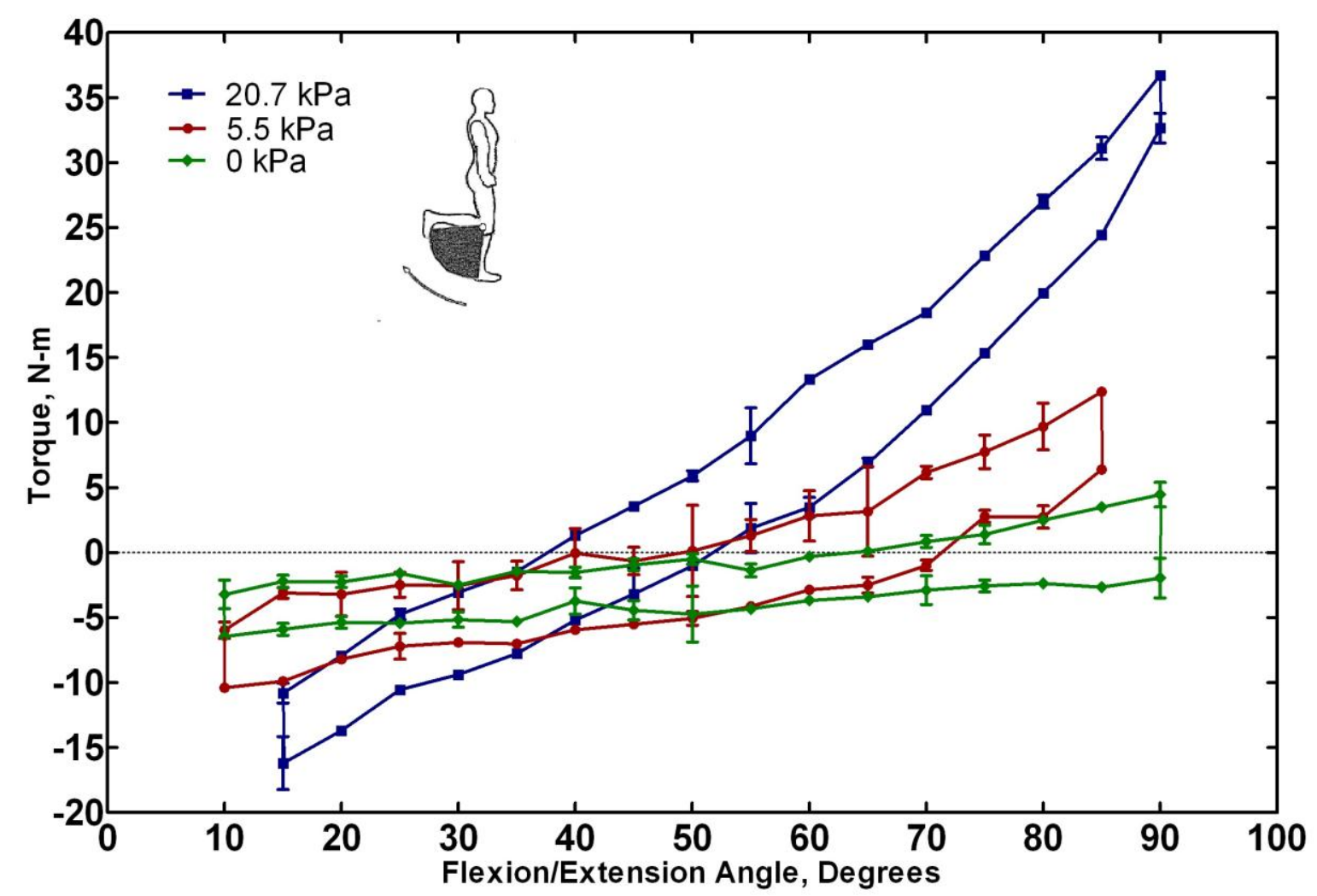

Figure 7. Knee flexion/extension torque vs. angle data with gravity effects removed

\section{Discussion}

This study created the first joint toque database for the S1034 PPA. It also provided researchers an opportunity to refine the testing techniques that will be used to test future space suit designs at MIT (for example, donning and doffing of the full suit was particularly complicated and took several hours, and a step-by-step process for doing this was created for future use).

Limitations of the robotic testing system were also identified during the course of this study. A hydraulic leak in the robot's thigh rotation joint was discovered, which forced researchers to operate the system at $10350 \mathrm{kPa}(1500$ psi) hydraulic pressure (well below its designed level of $20600 \mathrm{kPa}$, or $3000 \mathrm{psi}$ ) to minimize leakage of hydraulic fluid on the suit. Since testing, the hydraulic fluid leak has been fixed. Greater hydraulic pressure will increase the responsiveness of the robot to computer controlled inputs; enabling larger range of motion envelopes (should they be desired). Secondly, three of the four test cases (elbow flexion/extension being the only exception) were tested using manual control inputs only because of limitations in both suit flexibility (leading testers to be overly cautious about damaging the suit due to over-extension) and availability (leading to an accelerated test schedule, preventing proper calibration of the RSST's automatic movement controls for each joint). Manual control of the robot did produce consistent data, but this data was noisier than the data produced by movements generated by a sinusoidal input function. Additionally, only one degree-of-freedom (DOF) tests were conducted due to time constraints. Multiple DOF tests, with more natural human motions, would have been preferred as they are more representative of the actual intended use of the suit.

\section{Conclusion and Future Work}

In addition to providing insight into the mobility characteristics of the S1034 PPA, this study highlights the specific testing capabilities and advantages of robotic space suit testing. Advantages of robotic suit testing include: the capability to test suits in their integrated, pressurized and fully occupied state; the capability to articulate suits through complete ranges of motion in an anthropometrically realistic and repeatable manner (including natural 
human motions not limited to $1 \mathrm{DOF}$ ); and the capability to collect torque and angle measurements with precision and with low uncertainty. Though this system is not without flaws (as highlighted in Section I) it stands as a robust and precise tool for future pressure garment testing, and provides certain capabilities that are lacking in other torque testing methods.

While robotic testing stands as the best current option for quantifying pressure suit mobility characteristics, an ideal method to improve future pressure suit testing would be to create a skintight, lightweight, non-invasive, instrumented garment that can be worn by a suit subject on the inside of a pressurized suit. This garment would measure in real time the human's joint angles, which could be compared with external real-time measurements of the suit angles. As well, the garment could measure muscle activity through EMG to quantify the actual torques exerted by the subject's muscles, and it could measure the pressure profile of the interactions between the subject and the pressure suit. This garment could be worn by multiple subjects to obtain values for different anthropometries. This type of advanced instrumented garment would provide the greatest insight into the actual joint torques felt by human subjects.

Robotic space suit testing is the closest current approximation to this wearable garment. It accomplishes most of the same goals, with the differences noted in Section I. While it is not exactly of human anthropometry, it has the benefit of performing identical, repeatable joint motions, and it eliminates the uncertainty associated with human test subjects. Additional studies are warranted to quantify any robotic deviation from a human standard. Ultimately, a focused joint torque quantification project, either using the RSST or the advanced garment described above, combined with multiple iterations of joint design, could lead to drastic reduction of joint torques for future space suits.

\section{Acknowledgments}

The authors would like to thank the following people for their contributions to this research effort: Roedolph Opperman, Justin Kaderka, Dave Robertson, Todd Billings, and Dick Perdichizzi (MIT); and Dan Barry and Steve Koneczny (David Clark Company).

\section{References}

${ }^{1}$ Frazer, A.L., Pitts, B.M., Schmidt, P.B., Hoffman, J.A., Newman, D.J., “Astronaut Performance: Implications for Future Space Suit Design". International Astronautical Congress, 2002.

${ }^{2}$ Clark, D.M. "Some Practical Considerations in Pressure Suit Development." Proceedings of the Omni-Environment Full Pressure Suit Symposium. February, 1955.

${ }^{3}$ Holschuh, B., Waldie, J., Hoffman, J., Newman, D. "Characterization of Structural, Volume and Pressure Components to Space Suit Joint Rigidity.” 39th International Conference on Environmental Systems, SAE Paper 2009-01-2535. July 2009.

${ }^{4}$ Harris, G.L., The Origins and Technology of the Advanced Extravehicular Space Suit, AAS History Series, Vol. 24, 2001

${ }^{5}$ Bowen, J.D. "Evaluation of Manned Orbiting Laboratory Design Definition Pressure Garments." AMRL-TR-66-235. July, 1968.

${ }^{6}$ Lutz, C.C. "Mobility Characteristics, Apollo Block II A6L Pressure Garment Assembly." NASA Crew Systems Division, August 1967.

${ }^{7}$ Dionne, S. (1991). “AX-5, Mk III, and Shuttle Space Suit Comparison Test Summary." 91-SAE/SD-004, NASA Ames Research Center, Moffett Field, CA.

${ }^{8}$ Braden, Jeffrey R., and Akin, David L., "Development and Testing Update on the MX-2 Neutral Buoyancy Space Suit Analogue.” 34th International Conference on Environmental Systems, SAE Paper 04ICES-235, July 2004.

${ }^{9}$ Kaderka, J.D., Hoffman, J.A., Newman, D.J., "Enhanced Planetary Surface EVA Mobility for Space Colonization". To be presented at the 41st International Conference on Environmental System, July 2011.

${ }^{10}$ Matty, J.E., Aitchison, L., "A Method for and Issues Associated with the Determination of Space Suit Joint Requirements." $39^{\text {th }}$ International Conference on Environmental Systems, SAE Paper 2009-01-2537, July 2009.

${ }^{11}$ Schmidt, P.B., Newman, D.J., Hodgson, E., "Modeling Space Suit Mobility: Applications to Design and Operations." 31 st International Conference on Environmental Systems, SAE Paper 01ICES-115, July 2001.

${ }^{12}$ Morgan, D.A., Wilmington, R.P, Pandya, A.K., Maida, J.C., Demel, K.J. "Comparison of Extravehicular Mobility Unit (EMU) Suited and Unsuited Isolated Joint Strength Measurements.” NASA Technical Paper 3613, June 1996.

${ }^{13}$ Schmidt, P, "An Investigation of Space Suit Mobility with Applications to EVA Operations." Ph.D. Thesis, Massachusetts Institute of Technology, 2001.

${ }^{14}$ Matty, J.E., "Results and Analysis from Space Suit Joint Torque Testing." $40^{\text {th }}$ International Conference on Environmental Systems, AIAA Paper 2010-6211, July 2010.

${ }_{16}^{15}$ Polmar, N., "Spyplane: The U-2 History Declassified." MBI Publishing, 2001.

${ }^{16}$ O'Leary, M., Schulzinger, E., "Black Magic: America's Spyplanes: SR-71 and U-2." Motorbooks International, 1989. 